\title{
La longue et sinueuse route vers l'amélioration des taux de vaccination : communication des pratiques exemplaires au Canada
}

\author{
MacDonald, $\mathrm{NE}^{1 *}$
}

\section{Résumé}

Les vaccins sont parmi les façons les plus efficaces de réduire la mortalité infantile. Toutefois, dans un rapport de 2013 de l'UNICEF comparant les taux d'adoption des vaccins de la petite enfance, le Canada se trouve malheureusement au $28^{\mathrm{e}}$ rang des 29 pays à revenu élevé. Comme on peut le voir dans le budget fédéral de 2016, qui souligne l'importance d'améliorer l'accès à l'immunisation, des travaux sont en cours en vue de régler ce problème. De nombreuses mesures peuvent être prises afin d'améliorer l'adoption des vaccins, comme la détection et la meilleure compréhension des facteurs au niveau des personnes et des programmes qui sous-tendent le retard à recevoir des vaccins ou au refus de ces derniers. Cependant, il est difficile de trouver des données probantes et de s'assurer de leur pertinence dans le contexte canadien. On doit établir des ressources ciblées afin d'aborder la complexité de l'immunisation, tout au long du continuum, de la fabrication des vaccins jusqu'à leur adoption par les patients. Bien qu'il existe beaucoup de renseignements utiles pour le Canada, ils n'ont pas été regroupés en un endroit " de référence » et ils ne sont pas organisés. Le Canada a besoin d'une plateforme solide, facilement accessible et conviviale pour communiquer aux professionnels de la santé, aux parents et aux patients les mesures qui fonctionnent en matière d'immunisation. Cette plateforme serait un grand pas vers la facilitation de l'acceptation des vaccins au Canada.

\section{Affiliation}

${ }^{1}$ Département de pédiatrie maladies infectieuses, Faculté de médecine, Université Dalhousie, Halifax (Nouvelle-Écosse)

*Correspondance : noni. macdonald@dal.ca

Citation proposée : MacDonald, NE. La longue et sinueuse route vers l'amélioration des taux de vaccination : communication des pratiques exemplaires au Canada. Relevé des maladies transmissibles au Canada 2016;42(12):270-3. https://doi.org/10.14745/ccdr.v42i12a01f

\section{Introduction}

Partout dans le monde, les vaccins sont d'importants outils de réduction de la mortalité infantile. Ils sont si importants qu'en 2010, le Plan d'action mondial pour les vaccins a élaboré une vision pour la Décennie de la vaccination en vue de mettre en place un cadre de travail pour prévenir des millions de décès d'ici 2020, grâce à un accès plus équitable aux vaccins existants, pour toutes les personnes de toutes les collectivités. Ce plan a été approuvé par les 194 États membres de l'Assemblée mondiale de la santé (y compris le Canada) en mai 2012 (1). Toutefois, malgré le fait que les vaccins ont éradiqué la variole, qu'ils viendraient bientôt à bout de la poliomyélite, qu'ils ont récemment eu le mérite d'avoir éliminé la rougeole indigène en Amérique et qu'ils sont étayés par un important ensemble de données scientifiques de qualité supérieure démontrant l'importance de la vaccination tout au long de la vie, l'immunisation des nourrissons, des enfants, des adolescents et des adultes n'est ni acceptée volontiers ni exigée par tous.

\section{Couverture vaccinale}

Nous sommes maintenant à mi-chemin de la Décennie de la vaccination, mais le Canada se classe à un triste $28^{\mathrm{e}}$ rang des 29 pays à revenu élevé dans le Bilan Innocenti de 2013 de l'Office of Research de I'UNICEF, qui compare les taux d'adoption des vaccins de la petite enfance entre tous les pays (2). Comme de nombreuses provinces et de nombreux territoires n'ont pas de registre fiable d'immunisation, certains experts ne sont pas d'accord avec le taux canadien déclaré de $84 \%$, mais aucun d'entre eux n'affirmerait que le Canada s'approche des $95 \%$ et plus déclarés par la Finlande et plusieurs autres pays, loin de là. L'objectif stratégique numéro 2 du Plan $d^{\prime}$ action mondial pour les vaccins affirme que les personnes et les collectivités comprennent l'utilité des vaccins et exigent l'immunisation en tant que droit et responsabilité (1). Peu importe que son taux d'immunisation dans la petite enfance soit précisément de $84 \%$ ou non, le Canada doit améliorer sa situation, en matière d'acceptation et de demande à l'égard des vaccins, non seulement en ce qui a trait aux vaccins de la petite enfance, mais aussi concernant la vaccination des adolescents et des adultes, notamment des femmes enceintes. Le cas d'embryopathie rubéolique signalé en Ontario à la fin de l'année 2015 révèle que des lacunes persistent, dans les programmes de protection contre la rubéole fœtale, ce qui peut mener à des conséquences tragiques pouvant durer toute la vie (3). 
L'amélioration de l'équité en matière d'accès à l'immunisation a été soulignée dans le budget fédéral du Canada de 2016, qui destine 25 millions de dollars sur plus de cinq ans, à partir de 2016-2017, pour que " l'Agence de la santé publique du Canada [mette] à jour les objectifs nationaux de couverture vaccinale et les cibles pour les maladies évitables par la vaccination, [améliore] la capacité du Canada à identifier les personnes sous-immunisées ou non immunisées et [crée] un programme ciblé pour améliorer l'accès à la vaccination et les taux de vaccination » (4). Les recommandations du Groupe de travail expert sur l'acceptation et l'adoption des vaccins fournissent une feuille de route à l'Agence de la santé publique du Canada (ASPC) afin d'aller de l'avant pour vaincre l'hésitation relative aux vaccins (Données non publiées. Dr Robert Pless, Agence de la santé publique du Canada).

\section{Traitement de l'hésitation relative aux vaccins}

L'hésitation relative aux vaccins, ou « un retard dans l'acceptation ou un refus de la vaccination malgré la disponibilité des services de vaccination » est un problème bien reconnu au Canada et à l'échelle mondiale (5). Malheureusement, s'assurer de l'acceptation en temps opportun des vaccins n'est pas tâche facile pour les programmes d'immunisation provinciaux et territoriaux ou les professionnels de la santé de première ligne. Se limiter à fournir de l'information au sujet des avantages des vaccins et des risques des maladies évitables par la vaccination ne suffit pas $(6,7)$. En accordant trop d'importance uniquement aux faits appuyant les avantages des vaccins et les risques des maladies, on peut exacerber l'hésitation et diminuer l'acceptation des vaccins en temps opportun (8). L'article publié par Ève Dubé et ses collègues met l'accent sur la complexité du problème de l'hésitation relative aux vaccins et offre des stratégies potentielles d'intervention et de prévention qui peuvent être utiles dans le contexte canadien (9). Malheureusement, il n'existe aucune intervention universelle qui permettrait de répondre à toutes les préoccupations au sujet de la complaisance, de la commodité et de l'hésitation à faire confiance, relativement aux vaccins, qui pourrait faire de l'acceptation de la vaccination la norme pour tous, à l'exception d'une petite minorité (probablement de moins d'un à deux pour cent) qui demeurerait de fermes détracteurs de la vaccination (8).

Tel que souligné par Dubé et d'autres, écouter et déterminer les facteurs sous-jacents du retard ou du refus sont des mesures essentielles à prendre au niveau des personnes et des programmes (9-11). Les stratégies de communication et d'intervention doivent être adaptées en vue de régler le problème. On doit prendre en compte les préoccupations particulières des parents et des patients, que ce soit à propos de la sécurité des vaccins, des maladies évitables par la vaccination, de la peur des aiguilles ou d'autres problèmes. Comme

I'hésitation relative aux vaccins varie d'un vaccin à l'autre et selon le contexte, le moment et l'endroit (8), la liste des « nouvelles» préoccupations qui surgissent peut sembler infinie et intimidante, en particulier pour les intervenants de première ligne, si les données probantes ne sont pas à portée de main ou si elles ne sont pas pertinentes pour le parent ou le patient adulte.
Il est souvent difficile de trouver rapidement des données probantes et de s'assurer de leur pertinence dans le contexte canadien. L'article du présent numéro sur les tenants et les aboutissants de différents produits du Comité consultatif national de l'immunisation (CCNI) offre d'utiles conseils qui permettront au Canada de progresser (12). Par exemple, on y trouve des énoncés du CCNI fondés sur des données probantes sur le site Web de l'ASPC (13), des résumés du CCNI publiés dans le Relevé des maladies transmissibles au Canada, comme la déclaration annuelle sur la vaccination antigrippale (14) et la mise à jour sur l'hépatite $A(15)$, ainsi que de l'information sur l'immunisation axée sur la pratique, notamment des chapitres sur chaque vaccin dans le Guide canadien d'immunisation (16). Ces ressources sont toutes utiles, régulièrement mises à jour, et l'on peut s'inscrire afin de recevoir automatiquement les mises à jour du CCNI et du Guide canadien d'immunisation (17).

Malheureusement, un article expliquant où trouver quels renseignements ne suffit pas. Des ressources ciblées abordant la complexité de l'immunisation, de la fabrication des vaccins jusqu'à la vaccination des patients, sont nécessaires. Les parents et les patients ont fait part de leurs préoccupations à différents points de ce continuum, de la production jusqu'à l'injection. Les inquiétudes à propos de la sécurité des vaccins sont courantes. Elles peuvent compromettre la confiance envers le système et augmenter l'hésitation relative aux vaccins. Le Canada dispose d'un système de sécurité des vaccins très solide, conçu afin d'assurer la qualité supérieure et la sécurité des vaccins. Ce pays fournit un système de vaccination de haute qualité pour offrir des services d'immunisation aux patients et un système de surveillance de haute qualité visant à détecter les effets indésirables à la suite de la vaccination et à déterminer s'ils sont liés au vaccin ou au programme de vaccination, et s'il est nécessaire de prendre des mesures. En fait, le Canada est un chef de file en matière de sécurité des vaccins. Cependant, de nombreux professionnels de la santé ne sont pas au courant de la solidité et de la portée du système de sécurité des vaccins. Le Comité des maladies infectieuses et d'immunisation de la Société canadienne de pédiatrie fournit un aperçu pratique du programme de sécurité des vaccins du Canada qui peut aider les intervenants de première ligne à mieux répondre aux questions des parents et des patients au sujet du système de sécurité des vaccins (18). L'importance du Réseau de cliniques d'immunisation spéciales partout au pays ne peut être sous-estimée. Le personnel de ces cliniques est composé de spécialistes des vaccins pour enfants et pour adultes qui possèdent une solide expérience des réponses aux effets indésirables complexes qui suivent une vaccination et qui peuvent contribuer à évaluer et à conseiller les personnes ayant subi un effet indésirable grave à la suite d'une vaccination.

\section{Accéder aux pratiques exemplaires}

Les articles du présent numéro ne font qu'effleurer les besoins des gestionnaires de programmes et des travailleurs de la santé de première ligne en matière de données probantes et d'information. Bien que beaucoup de renseignements pertinents pour le Canada soient accessibles, ils n'ont pas été regroupés en un endroit " de référence » et ils ne sont pas organisés. Par exemple, de nombreux intervenants de première ligne peuvent ne pas connaître les conseils que la Société 
canadienne de pédiatrie adresse aux parents sur les risques et les responsabilités relatifs à la vaccination (19). De même, les gestionnaires de programmes d'immunisation ne connaissent probablement pas les lignes directrices du Bureau régional de I'Europe de l'Organisation mondiale de la Santé sur la façon de répondre en public aux détracteurs de la vaccination - une tâche que beaucoup trouvent très stressante (20). Même une connaissance de base de ces documents ne les rend pas plus accessibles sur Internet. De plus, il n'existe pas de façon simple de consulter un expert, lorsqu'on se trouve devant un casse-tête relatif à l'immunisation. Contrairement aux États-Unis, le Canada n'a pas de "coalition d'action pour l'immunisation », qui " s'efforce d'augmenter les taux d'immunisation et de prévenir les maladies, en créant et en distribuant des documents de formation aux professionnels de la santé et au public en vue d'améliorer la prestation de services d'immunisation sûrs et efficaces " [traduction] (21). Bien qu'Immunisation Canada soit un début, ce programme n'est ni financé ni équipé pour offrir la quantité de documents et les activités d'organisation requis, et n'a pas la capacité de répondre aux requêtes relatives à l'immunisation au Canada (22).

\section{Conclusion}

Le Canada pourrait être un chef de file, en matière de taux de vaccination des enfants, des adolescents, des adultes et des personnes âgées. Nous avons besoin d'une plateforme plus solide, facilement accessible et conviviale pour les professionnels de la santé, les parents et les patients afin qu'ils échangent sur les mesures efficaces en matière d'immunisation. II ne suffit pas d'offrir de l'information en vrac en soutien à une priorité mondiale. Une plateforme de référence offrant des ressources utiles et des données probantes en matière de pratiques exemplaires serait une avancée majeure dans le soutien à I'amélioration de l'acceptation de la vaccination au Canada.

\section{Références}

1. Organisation mondiale de la Santé (OMS). Plan d'action mondial pour les vaccins 2011-2020 [Internet]. Geneva: OMS; 2012 [consulté le 25 oct 2016]. Disponible à l'adresse : http://www.who.int/immunization/global_vaccine_action_ plan/fr/.

2. UNICEF Centre de recherche. Le bien-être des enfants dans les pays riches: Vue d'ensemble comparative. Bilan Innocenti 11 [Internet]. Florence, Italy: UNICEF; 2013 [consulté le 24 oct 2016]. Disponible à l'adresse : www.unicef-irc.org/ publications/pdf/rc11_fre.pdf.

3. Gouvernement du Canada. Rapport hebdomadaire de surveillance de la rougeole et de la rubéole : DU 14 février au 20 février 2016 (Semaine 7) [Internet]. Ottawa, ON: ASPC; 2016 [consulté le 24 oct 2016]. Disponible à l'adresse : http://healthycanadians.gc.ca/publications/ diseases-conditions-maladies-affections/measles-rubella2016-07-surveillance-rougeole-rubeole/index-fra.php.

4. Gouvernement du Canada [Internet]. Assurer la croissance de la classe moyenne. Chapitre 5. Une Canada inclusive et équitable. Ottawa: Gouverenment du Canada; 2016 [mis à jour le 22 mars 2016; consulté le 24 oct 2016]. Disponible à
I'adresse : http://www.budget.gc.ca/2016/docs/plan/ch5-fr. html.

5. Strategic Advisory Group of Experts on Immunization (SAGE). Report of the SAGE Working Group on Vaccine Hesitancy [Internet]. Geneva: WHO; 2014 [consulté le 24 oct 2016]. Disponible à l'adresse : www.who.int/immunization/ sage/meetings/2014/october/SAGE_working_group_ revised_report_vaccine_hesitancy.pdf?ua $=1$.

6. Jarrett $\mathrm{C}$, Wilson R, O'Leary M, Eckersberger E, Larson $\mathrm{HJ}$ and the SAGE Working Group on Vaccine Hesitancy. Strategies for addressing vaccine hesitancy: A systematic review. Vaccine [Internet]. 2015;33(34):4180-90. Disponible à l'adresse : http://ac.els-cdn.com/S0264410X15005046/1s2.0-S0264410X15005046-main.pdf?_tid=12c0d644-866711e6-b708-00000aacb35f\&acdnat=1475168973_2ff75a5cfd0 b5a11b01d61a7669dcda8.

7. Dubé E, Gagnon D, MacDonald NE and the SAGE Working Group on Vaccine Hesitancy. Strategies for addressing vaccine hesitancy: Review of published reviews. Vaccine [Internet]. 2015;33:4191-203. Disponible à l'adresse : http://ac.els-cdn.com/S0264410X15005058/1-s2.0S0264410X15005058-main.pdf?_tid=2fad48be-8667-11e6b99c-00000aacb361\&acdnat $=1475169022 \_993 a 4854$ e65a7f 9670912 ed86d6ac545.

8. MacDonald NE and the SAGE Working Group on Vaccine Hesitancy. Vaccine hesitancy: Definition, scope and determinants. Vaccine [Internet]. 2015;33:4161-4. Available from: http://ac.els-cdn.com/S0264410X15005009/1-s2.0S0264410X15005009-main.pdf?_tid=9bccbd66-8669-11e68a7e-00000aab0f01\&acdnat=1475170062_eee93e145066ae 4d4979ef4b566dac94.

9. Dubé E, Bettinger JA, Fisher WA, Hilderman T. Acceptation et refus de la vaccination et réticence face à cette pratique au Canada : défis et approches proposées. Relevé des maladies transmissibles au Canada [Internet]. 2016;42(12):274-9. Disponible à l'adresse : http://www.phacaspc.gc.ca/publicat/ccdr-rmtc/16vol42/dr-rm42-12/ar-02-fra. php.

10. Goldstein S, MacDonald NE, Guirguis $S$ and the SAGE Working Group on Vaccine Hesitancy. Health communication and vaccine hesitancy. Vaccine [Internet]. 2015;33:42124. Disponible à l'adresse : http://ac.els-cdn.com/ S0264410X1500506X/1-s2.0-S0264410X1500506X-main. pdf?_tid=48776cda-866c-11e6-8737-00000aacb35f\&acdnat =1475171211_466521448f611aeaf4a32a7ff29c90a1.

11. Butler R, MacDonald NE and the SAGE Working Group on Vaccine Hesitancy. Diagnosing the determinants of vaccine hesitancy in specific subgroups: The Guide to Tailoring Immunization Programmes (TIP). Vaccine [Internet]. 2015;33: 4176-9. Disponible à l'adresse : http://ac.els-cdn.com/ S0264410X15005022/1-s2.0-S0264410X15005022-main. pdf?_tid=335e320c-866c-11e6-b3ba-00000aacb35e\&acdnat =1475171175_783d26eb7165aa0b92e6686d404ef8c3.

12. Desai $S$, Tunis $M$, Stirling $R$, Jensen $C$, Ismail $S$, Baclic $O$, Lerch R. Produits d'orientation en matière d'immunisation : différents niveaux de détail pour différents usages. Relevé des maladies transmissibles au Canada. 2016;42(12):2804. Disponible à l'adresse : http://www.phac-aspc.gc.ca/ publicat/ccdr-rmtc/16vol42/dr-rm42-12/ar-03-fra.php. 
13. Agence de la santé publique du Canada [Internet]. Comité consultatif national de l'immunisation (CCNI) Recommandations, déclarations et mises à jour. Ottawa: ASPC; 2016 [mise à jour le 17 oct 2016; consulté le 24 oct 2016]. Disponible à l'adresse : http://www.phac-aspc.gc.ca/ naci-ccni/index-fra.php.

14. Gemmill I, Zhao $L$ et Cochrane $L$ au nom du Comité consultatif national de l'immunisation (CCNI). Résumé de la Déclaration sur la vaccination antigrippale pour la saison 2016-2017 du Comité consultatif national de l'immunisation (CCNI). Relevé des maladies transmissibles au Canada 2016;42:210-4. Disponible à l'adresse : http://www.phacaspc.gc.ca/publicat/ccdr-rmtc/16vol42/dr-rm42-9/ar-06-fra. php.

15. Henry B, Baclic $O$ au nom du Comité consultatif national de l'immunisation (CCNI). Résumé de la mise à jour de la déclaration du Comité consultatif national de l'immunisation (CCNI) sur l'usage recommandé du vaccin contre I'hépatite A. Relevé des maladies transmissibles au Canada 2016;42:215-8. Disponible à l'adresse : http://www. phac-aspc.gc.ca/publicat/ccdr-rmtc/16vol42/dr-rm42-9/ar07-fra.php.

16. Agence de la santé publique du Canada [Internet]. Guide canadien d'immunisation. Ottawa: ASPC; 2016 [mis à jour le 01 sept 2016; consulté le 24 oct 2016]. Disponible à I'adresse : http://healthycanadians.gc.ca/healthy-living-viesaine/immunization-immunisation/canadian-immunizationguide-canadien-immunisation/index-fra.php.

17. Gouvernement du Canada [Internet]. Mise à jour du Guide canadien d'immunisation : Liste de diffusion. [mis à jour le 13 mai 2014; consulté le 24 oct 2016]. Disponible à l'adresse
: http://healthycanadians.gc.ca/healthy-living-vie-saine/ immunization-immunisation/canadian-immunization-guidecanadien-immunisation/email-subscription-abonnementcourriel-fra.php.

18. Société canadienne de pédiatrie Comité des maladies infectieuses et d'immunisation. Auteurs principaux: MacDonald NE et Law BJ(le 15 novembre 2016). Le système d'innocuité vaccinale canadien en huit étapes : des notions pour les travailleurs de la santé (Point de pratique). Disponible à l'adresse : http://www.cps.ca/fr/documents/ position/systeme-innocuite-vaccinale.

19. Société canadienne de pédiatrie (SCP) [Internet]. Les parents qui choisissent de ne pas faire vacciner leur enfant : les risques et les responsabilités. Ottawa: CPS; 2016 [mis à jour sept 2016; consulté le 24 oct 2016]. Disponible à l'adresse : http://www.soinsdenosenfants.cps.ca/handouts/whenparents-choose-not-to-vaccinate-risks-and-responsibilities.

20. Organisation mondiale de la Santé (OMS). Best practice guidance: How to respond to vocal vaccine deniers in public [Internet]. Geneva: WHO; 2016 [cited 2016 Oct 24]. Disponible à l'adresse : http://www.euro.who.int/en/healthtopics/disease-prevention/vaccines-and-immunization/ publications/2016/best-practice-guidance-how-to-respondto-vocal-vaccine-deniers-in-public-2016.

21. Immunization Action Coalition [Internet]. Saint Paul, Minnesota: IAC; 2016 [consulté le 24 oct 2016]. Disponible à I'adresse : http://www.immunize.org/.

22. Immunisation Canada [Internet]. Ottawa: CPHA; 2016 [mise à jour le 22 sept 2016; consulté le 24 oct 2016]. Disponible à l'adresse : http://www.immunize.ca/fr/default.aspx.

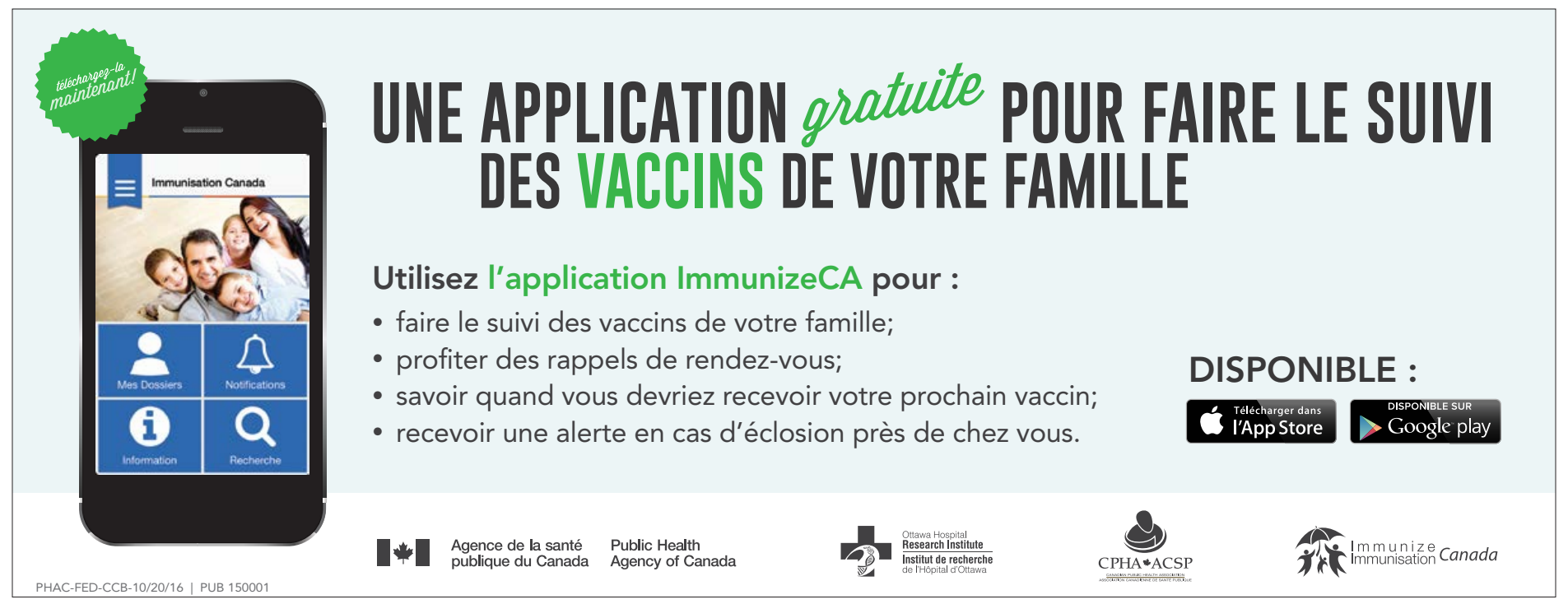

